This item was submitted to Loughborough's Research Repository by the author.

Items in Figshare are protected by copyright, with all rights reserved, unless otherwise indicated.

\title{
Non-occupational sitting and mental well-being in employed adults
}

PLEASE CITE THE PUBLISHED VERSION

http://dx.doi.org/10.1007/s12160-011-9320-y

\section{PUBLISHER}

Springer-Verlag (@ The Society of Behavioral Medicine 2011)

\section{VERSION}

AM (Accepted Manuscript)

\section{PUBLISHER STATEMENT}

This work is made available according to the conditions of the Creative Commons Attribution-NonCommercialNoDerivatives 4.0 International (CC BY-NC-ND 4.0) licence. Full details of this licence are available at: https://creativecommons.org/licenses/by-nc-nd/4.0/

\section{LICENCE}

CC BY-NC-ND 4.0

\section{REPOSITORY RECORD}

Atkin, Andrew J., Emma J. Adams, Fiona C. Bull, and Stuart J.H. Biddle. 2019. "Non-occupational Sitting and Mental Well-being in Employed Adults". figshare. https://hdl.handle.net/2134/19256. 


\begin{abstract}
Background: Emerging evidence suggests that sedentary behaviour may be adversely associated with physical health, but few studies have examined the association with mental well-being. Purpose: This study examined the association of four non-occupational sedentary behaviours, individually and in total, with mental well-being in employed adults. Methods: Baseline data from the evaluation of Well@Work, a national workplace health promotion project conducted in the UK, were used. Participants self-reported sitting time whilst watching television, using a computer, socialising and travelling by motorised transport. Mental well-being was assessed by the 12-item version of the General Health Questionnaire (GHQ-12). Analyses were conducted using multiple linear regression. Results: In models adjusted for multiple confounders, TV viewing, computer use, and total non-occupational sitting time were adversely associated with GHQ-12 assessed mental well-being in women. No statistically significant associations were observed in men. Conclusions: Nonoccupational sitting is adversely associated with mental well-being in women but not men. Keywords: sedentary behaviour, sitting time, mental well-being, effect modification
\end{abstract}




\section{Non-Occupational Sitting and Mental Well-Being in Employed Adults}

Seminal work by Jeremy Morris in the 1950s showed that high occupational sitting time was associated with adverse cardiovascular health [1]. However, it was not until the late 1990s that research developed rapidly concerning the likely negative health consequences of excessive sitting, and much of the impetus for this was due to ubiquitous new technologies in the home and at work [2 3]. Research with young people and adults is now showing that sedentary behaviour, when assessed by self-report or by objective monitor, is associated with higher weight status [4 5], markers of adverse metabolic health [6], and even mortality [7 8]. However, less has been reported on associations with mental health.

Mental ill health contributes substantially to the global burden of disease [9]. Projections suggest that unipolar depressive disorder, for example, will be one of the leading causes of the burden of disease by 2030, alongside ischemic heart disease and HIV/AIDS [10]. Research to identify factors that may increase the risk of mental ill-health is, therefore, a public health priority. The role of physical activity in promoting mental health has been well studied [11]. In recent years, however, there has been growing interest in the influence of behaviours from across the energy expenditure continuum on health and well-being [12 13]. One factor that has contributed to this movement is the recognition of sedentary behaviour as a distinct domain of behaviour, which may pose a risk to health independently of physical activity. There is a need, therefore, to examine whether sedentary behaviours are associated with mental health. This line of research may lead to the identification of new risk factors for mental ill-health and subsequently to the development of effective prevention and treatment strategies, either adjunctive to or independent of existing practices.

Emerging evidence is suggestive of an inverse association between sedentary behaviour and mental health in adults. For example, in a review of seven observational studies, risk of depression was elevated at higher levels of sedentary behaviour when 
measured primarily as some form of self-reported screen time, with one study showing a similar association when measuring sedentary time using an accelerometer [14]. In Scottish adults, Hamer et al. [15] studied the association of recreational screen-time with mental wellbeing, assessed using the 12-item version of the General Health Questionnaire (GHQ-12). The GHQ-12 is a measure of psychological distress, which has been shown to be highly associated with various psychological disorders, such as depression and anxiety [16]. The highest group for recreational screen-time (>4 hours/day) had an increase in GHQ-12 score of 0.28 (95\% CI $0.05,0.51)$ in comparison to those with low screen time ( 2 hours/day or less) after controlling for confounders, including physical activity.

Most studies, as shown in the review by Teychenne et al. [14], assess sedentary behaviour with reference to recreational screen time. However, one study of disadvantaged Australian women [17] reported that self-reported total sitting time, in addition to computer time and screen-time, was adversely associated with the risk of depression. However, some research has highlighted that not all sedentary behaviours may have the same type of association with mental health, with evidence for better mental health from computer / internet use [18]. The mechanisms through which sedentary behaviours may impact upon mental health are not well understood. There is, for example, a lack of consensus as to whether internet / computer use impairs or improves communication and social networks [18 19]. Sedentary behaviour may be detrimental to health through displacement of physical activity, but this hypothesis has not been widely explored in the context of mental health. In addition, psychological factors, such as social competence or motivation, may act as either mediators or moderators of the association with mental health. Taken together, these findings indicate the need to examine the influence of a wider range of sedentary behaviours than screen-time and total sitting on mental health. Some sedentary behaviours, such as use of motorised travel and sedentary socialising, have been understudied for their associations with 
physical and mental health. The current study, therefore, examined the association of four different non-occupational sedentary behaviours, as well as total non-occupational sitting, with mental well-being in a large sample of working adults.

\section{Methods}

\section{Participants and procedure}

From July 2005 to March 2006 a baseline survey was conducted in 32 workplaces from 9 regions of England as part of the evaluation of the national Well@Work project. All employees in all organisations $(n=10353)$ were invited to participate. The survey was available as a paper version or an on-line web-based survey. In organisations using the webbased survey, employees were invited to participate and sent the web link via email or electronic newsletter. Distribution of the paper version of the survey varied across organisations but in each case the survey was provided with a covering letter, the information sheet and a pre-paid return envelope. A second copy of the survey or a reminder e-mail was distributed two to three weeks after the initial survey distribution to non-responders. During the survey period, employees were prompted to complete the survey in a variety of ways including at team/department meetings, through staff presentations, via line managers, emails, notices in organisational newsletters and posters displayed in the workplace. A prize draw incentive to complete the survey was offered. The baseline survey took place before any interventions were delivered. A total of 3490 employees responded to the survey giving a response rate of $34 \%$ (range 16\%-51\% across organisations). All employees provided consent to participate in the survey. Further details about the participating organisations and the methodology of the underlying evaluation are available in the Well@Work evaluation report [20].

\section{Sitting time}


Participants self reported usual time spent sitting (hours / minutes per day) in four domains of sedentary behaviour on work and non-work days, using items that have been previously validated where available [21] and new items developed specifically for the current study. New items were developed because, at the time of data collection, no previously validated tools with low participant burden were identified that adequately captured the exposures of interest for the evaluation study. Sedentary behaviour domains were travel (e.g. motorised travel by car, train, or bus); watching TV (including video / DVD); using a computer (including playing video games); and social sedentary activities (e.g. sitting whilst talking with friends, listening to music, playing seated games). Example item: “Thinking about a usual week, on a typical work and non-work day, how much time do you usually spend sitting using a computer (not for job related work) or playing video games". Weighted mean $(5 \mathrm{x}$ work $+2 \mathrm{x}$ non-work / 7) sitting time per day for each domain of sedentary behaviour was calculated. Total non-occupational sitting time per day was calculated as the sum of the four domain specific sitting time variables.

\section{Mental well-being}

Mental health was assessed using the 12-item version of the General Health Questionnaire (GHQ-12), which is a measure of psychological distress devised for population studies [16]. The questionnaire inquires about general level of happiness, experience of depressive and anxiety symptoms, and sleep disturbance during the past 4 weeks.

Interpretation of the answers is based on a 4-point response scale scored using a bimodal method (symptom present: not at all =0, same as usual =0, more than usual $=1$, and much more than usual =1). The GHQ-12 is a well validated instrument and has been strongly associated with various psychological disorders, such as depression and anxiety [15]. A total score was computed across the 12 items with a higher score (maximum 12) indicating greater psychological distress. 


\section{Covariates}

Participants self-reported date of birth, height and weight (used to calculate body mass index (BMI, weight $(\mathrm{kg}) /$ height $\left.(\mathrm{m})^{2}\right)$, marital status, level of education, household income, smoking habits, fruit and vegetable intake, alcohol consumption, and physical activity (occupational / leisure-time / active travel [21 22]. Detailed description of the items used to assess covariates can be found elsewhere [20].

\section{Statistical analysis}

Domain specific sitting time variables were categorised into 4 groups. TV viewing time was grouped as $<60,60-120,120-180$, and $>180$ minutes / day. Computer use, social sitting and travel-related sitting were grouped separately into <30, 30-60, 60-90, and >90 minutes / day. Total non-occupational sitting time was categorised into 4 groups; $<3,3-5,5-$ 7, and $>7$ hours / day. Analysis of variance and chi-square tests were used to test for differences in demographic, behavioural and anthropometric characteristics across total nonoccupational sitting time groups. Associations between domain specific and total nonoccupational sitting and GHQ-12 scores were examined using general linear models. Preliminary analyses indicated that associations between sitting time variables and mental well-being differed by gender, thus results are presented separately for men and women. Three models were fitted, which included (1) adjustment for age only (model 1); (2) further adjustment for marital status (single, partner non-cohabiting, partner cohabiting, separated / divorced, widowed), education (no qualifications, GCSE / other, A-level / degree), household income (unknown / prefer not to say, <£20800, £20800-£41600, >£41600 per year), smoking (never, former, current), BMI $(<25,25-30,>30)$, alcohol consumption (never, monthly or less,

2-4 times / month, 2-3 times / week, 4+ times / week), and daily fruit and vegetable intake (< 5, >5 servings / day) (model 2); and (3) further adjustment for occupational (not at all physically active, not very physically active, fairly physically active, very physically active) 
and leisure-time physical activity level ( $<60,60-180,180-300,>300$ minutes / week). This modelling strategy allowed us to control for potential confounding and examine whether associations between sitting time and GHQ-12 scores were independent of participation in physical activity. In additional analyses, logistic regression was used to examine the association between sitting time and psychological distress, using an established cut-point (GHQ-12 score $\geq 4$ ) to define distress. Robust (Huber-White sandwich estimates) standard errors were used to take account of clustering (non-independence between participants from the same workplace) in the computation of $95 \%$ confidence intervals and P-values. Analyses were performed using the complex samples procedure in SPSS, version 16, and alpha was set at 0.05 .

\section{Results}

Overall, the mean (SD) age of participants was 40.4 (11.0) years and 58\% were female. Total non-occupational sitting time (mean (SD)) for the entire sample was 300.2 (155.6) minutes per day. The mean (SD) GHQ-12 score for the whole sample was 1.8 (2.6). Participant characteristics with reference to total non-occupational sitting time are provided in Table 1. Participants in the highest group for total sedentary time were more likely to be male, younger, less likely to meet physical activity and fruit and vegetable guidelines, had higher BMI and were more likely to be current smokers.

In women, TV viewing, computer use, and total sitting were positively associated with GHQ-12 scores (Table 2). Associations were attenuated slightly after adjustment for multiple covariates, but remained statistically significant (model 2). Further adjustment for physical activity (model 3) produced minimal changes in $\beta$ coefficients, suggesting physical activity is a weak confounder of the association. In the fully adjusted model (model 3), women with the highest levels of TV viewing, computer use, and total sitting time had increased GHQ-12 scores of 0.41 (95\% CI 0.08, 0.75), 0.64 (95\% CI 0.34, 0.93) and 0.51 (95\% 
CI $0.22,0.80)$ respectively compared with participants in the lowest groups. For each additional hour of TV viewing, computer use and total non-occupational sitting the mean GHQ-12 score increased by 0.11 (95\% CI 0.01, 0.20), 0.12 (95\% CI 0.02, 0.21) and 0.10 (95\% CI $0.06,0.14$ ) respectively (model 3). A positive association that was of borderline statistical significance was observed for travel-related sitting time and GHQ-12 scores. Women in the highest group for travel-related sitting had increased GHQ-12 scores of 0.51 (95\% CI -0.02, 1.04; model 3) compared with those in the lowest group.

In men, TV viewing, travel-related and total sitting were not found to be associated with GHQ-12 scores in any of the statistical models tested (Table 2). A positive association was observed for computer use and GHQ-12 scores. In the fully adjusted model (model 3), men in the highest group for computer use had increased GHQ-12 scores of 0.28 (95\% CI $0.03,0.53)$ compared with those in the lowest group. For each additional hour of computer use the mean GHQ-12 score increased by 0.06 (95\% CI 0.00, 0.12) (model 3). There was some evidence for a negative association between social sitting time and GHQ-12 scores. In the fully adjusted model (model 3), men in the highest group for social-sitting had reduced GHQ-12 scores of -0.24 (95\% CI $-0.65,0.18)$ compared with those in the lowest group, but findings were not statistically significant.

In logistic regression analyses, men and women in the highest groups for computer use had greater risk of psychological distress (defined as GHQ-12 score $\geq 4$ ) compared to those in the lowest group; women OR 1.70 (95\% CI 1.19 2.44), men OR 1.80 (95\% CI 1.21, 2.67). Higher levels of sedentary behaviour were associated with increased odds of psychological distress for each of the remaining sedentary time variables, with the exception of social sitting in men which showed a negative association, but none of the associations attained statistical significance in adjusted models.

\section{Discussion}


The aim of this study was to examine the cross-sectional association of selected nonoccupational sedentary behaviours with mental well-being, assessed by GHQ-12. Findings indicate that self-reported TV viewing, computer use, and total non-occupational sitting time were adversely associated with mental well-being in women, independent of potential confounders including physical activity. In men, computer use only was found to be positively associated with GHQ-12 scores. The potential moderating effect of gender on associations between sedentary behaviour and mental well-being has not been reported previously. Further research in studies using prospective designs are required to provide more robust evidence on whether the observed associations are causal in nature.

The evidence base linking various indicators of sedentary behaviour with physical health has expanded rapidly in recent years [ 345 4 ] but much less research has been conducted on the potentially adverse impact of sedentary behaviour on psychological well-being. Television viewing and screen-based entertainment were cross-sectionally associated with GHQ-12 assessed mental well-being, independent of confounding factors including leisuretime physical activity, in a representative sample of Scottish adults [15]. However, analyses were presented for men and women combined and the authors provide no comment on whether a potential interaction by gender was explored. In women only samples, screenbased sedentary behaviours, total self-reported sitting time and objectively assessed total sedentary time have been associated with depressive symptoms [23 24]. A gender difference in the health outcomes of sedentary behaviour has been observed previously, with findings consistent with our study that sedentary behaviour appears to pose a greater risk for women than men [3 4 5]. This may be the result of different patterns of sedentary behaviour between genders or, in the case of psychological well-being, reflect contrasting psychological mediators that underpin prolonged engagement in sedentary behaviour. Further research into the presence of, and mechanisms behind, a gender difference in the physical and 
psychological health outcomes of sedentary behaviour is required and will help to establish more clearly whether associations are causal.

Findings of the current study highlight the importance of assessing multiple sedentary behaviours when exploring possible relationships with psychological health outcomes. Not all of the sedentary behaviours examined were found to be related with mental well-being and where associations were identified they sometimes differed in their magnitude and direction. This is consistent with a recent review of the evidence. Teychenne et al. [14] reported that most of the research to date has noted a positive association between sedentary behaviour and risk of depression. However, those studies that included computer / internet use as the exposure variable were more likely to report null or even inverse associations with risk of depression. Whilst it is important to acknowledge that the current study examined general mental well-being rather than depression, which may exhibit different associations with sedentary behaviour, it is valuable to consider potential implications of these findings. For example, it may be that for the investigation of psychological health outcomes, use of 'combined' indicators of sedentary behaviour, such as 'screen-time' (TV viewing, computer / video game use) may be inappropriate, as the constituent behaviours of these indicators may be differentially associated with the outcomes under study. Moreover, the utility of objective monitoring devices (e.g. accelerometers) for the examination of associations between sedentary behaviour and psychological health requires further study, because these technologies assess only the total volume of sedentary behaviour and do not distinguish between the different behaviours that make-up total sedentary time.

Due to the cross sectional nature of this study, it is not possible to ascertain the direction of the association between sedentary behaviour and mental well-being (i.e. whether sedentary behaviour is a cause or consequence of poorer mental well-being). It is acknowledged that reverse causality is a potential explanation for the observed associations; 
those individuals with poorer mental health may be more inclined to engage in sedentary behaviours. Moreover, due to the episodic nature of some mental health conditions, it is possible that there is a cyclical and reciprocal association between sedentary behaviour and mental health that may lead to an inflated estimate of the association in cross-sectional analyses. At this point, it is important to acknowledge that this remains a new and emerging field of research. The findings highlighted in this study, and others [15], provide valuable preliminary evidence for the potential health risks of behaviours that are highly prevalent in contemporary society. The limitations highlighted above do not preclude the possibility that overall reductions in sedentary behaviour or breaking up prolonged periods of sitting may contribute towards the prevention and treatment of mental ill-health. Studies using prospective and experimental designs are required to establish the temporal sequence of the observed associations.

A number of routes through which sedentary behaviour may be causally linked with psychological well-being have been hypothesised. Typically, these have focused upon psychological rather than biological mediators of the association, which provide valuable explanatory insight but are prone to bias and limited for establishing temporal sequence. Biologically plausible explanations for the association between physical activity and depression, for example, have served to strengthen the case for a causal relationship [11]. The social isolation hypothesis proposes that prolonged engagement in sedentary behaviours, such as TV viewing or computer use, leads to breakdown of social support or communication networks which may lead to increased risk of psychological ill-health. However, this hypothesis is not supported by evidence indicating that computer use can lead to improved social networks and communication [18]. Thus, it may be that it is the purpose of the behaviour or the motivation to engage in it that is key in determining the relationship with psychological health, rather than the duration of participation. This may account for the trend 
towards a protective association between social sitting and mental well-being observed in men, as this is likely to be a behaviour motivated by a desire to interact and socialise with friends or family. An alternative route through which sedentary behaviour may impact upon mental health is through the displacement of physical activity, which has been shown to be beneficially associated with psychological well-being [11]. Evidence indicating that sedentary behaviours displace participation in physical activity, however, is mixed. During specific segments of the day it is possible that sedentary and active behaviours may compete directly for time allocation but across the whole day correlations between sedentary behaviour and physical activity typically are quite weak [25]. Further research using prospective designs, and examination of potential biological as well as psychological mediators, will help to determine if the association between sedentary behaviour and mental well-being is causal in nature.

A strength of the current study was the collection of data from a large, diverse sample of working adults. In addition, to our knowledge, this is the first study that has examined associations of numerous non-occupational sedentary behaviours, including social sitting and travel-related sitting, with mental well being. The majority of previous research on this subject has focused solely on TV viewing or other screen-based sedentary behaviours. Further, we obtained good quality measurements of and statistically controlled for a large number of potential confounding factors, including physical activity and fruit and vegetable intake.

Limitations of the current study are acknowledged. Firstly, findings are derived from cross sectional research, thus it is not possible to establish causality or the direction of the associations observed. Secondly, associations between sitting time variables and GHQ-12 scores were attenuated in multivariate models, indicating that variables added to statistical models confounded the observed associations. Residual confounding may, in part, account 
for the associations observed, due to incomplete adjustment as a result of measurement error or failure to adjust for unknown or unmeasured confounders. We were, for example, unable to adjust for the potentially confounding effect of physical function, as no measures of this construct were obtained. However, previous research exploring the association between screen-based sedentary behaviour and mental well-being reported that adjustment for physical function attenuated the observed association but did not negate it [15]. Lastly, it may be argued that the effect sizes observed in this study are small and therefore may not be clinically relevant. However, associations are comparable in magnitude to those reported in previous studies on this topic [15 26] and due to the high prevalence sedentary behaviour even small increases in risk may be of public health significance.

Findings of this study are consistent with and extend those of previous research indicating that sedentary behaviour may be an independent risk factor for physical and psychological health. The unique contribution of the current study is the observation that associations of domain-specific and total non-occupational sitting time with mental well being may be moderated by gender. Adverse associations with mental well being were observed for TV viewing, computer use and total non-occupational sitting in women, whilst computer use only was found to be adversely associated with mental well-being in men. Replication of these findings in studies using prospective designs is necessary.

Conflict of interest statement: The authors have no relevant conflicts of interest to disclose. 


\section{References}

1 Morris JN, Heady JA, Raffle PAB, et al. Coronary Heart-Disease and Physical Activity of Work. Lancet. 1953; 265: 1111-1120.

2 Owen N, Leslie E, Salmon J, et al. Environmental determinants of physical activity and sedentary behavior. Exerc Sport Sci Rev. 2000; 28(4): 153-158.

3 Owen N, Healy GN, Matthews CE, et al. Too much sitting: the population health science of sedentary behavior. Exerc Sport Sci Rev. 2010; 38(3): 105-113.

4 Marshall SJ, Ramirez E. Reducing sedentary behavior: A new paradigm in physical activity promotion. Am J Lifestyle Med. 2011; DOI: 10.1177/1559827610395487.

5 Tremblay MS, Colley RC, Saunders TJ, et al. Physiological and health implications of a sedentary lifestyle. Appl Physiol Nutr Metab. 2010; 35(6): 725-740.

6 Dunstan DW, Salmon J, Healy GN, et al. Association of television viewing with fasting and 2-h postchallenge plasma glucose levels in adults without diagnosed diabetes. Diabetes Care. 2007; 30(3): 516-522.

7 Dunstan DW, Barr EL, Healy GN, et al. Television viewing time and mortality: the Australian Diabetes, Obesity and Lifestyle Study (AusDiab). Circulation. 2010; 121(3): 384-391.

8 Katzmarzyk PT, Church TS, Craig CL, et al. Sitting time and mortality from all causes, cardiovascular disease, and cancer. Med Sci Sports Exerc. 2009; 41(5): 998-1005.

9 Prince M, Patel V, Saxena S, et al. No health without mental health. Lancet. 2007; 370(9590): 859-877. 
10 Mathers CD, Loncar D. Projections of global mortality and burden of disease from 2002 to 2030. PLoS Med. 2006; 3(11): e442.

11 Biddle SJH, Fox KR, Boutcher S. Physical activity and psychological well-being. London: Routledge, 2000.

12 Healy GN, Dunstan DW, Salmon J, et al. Objectively measured light-intensity physical activity is independently associated with 2-h plasma glucose. Diab Care. 2007; 30(6): 1384-1389.

13 Katzmarzyk PT. Physical activity, sedentary behavior, and health: paradigm paralysis or paradigm shift? Diabetes. 2010; 59(11): 2717-2725.

14 Teychenne M, Ball K, Salmon J. Sedentary behavior and depression among adults: a review. Int J Behav Med. 2010; 17(4): 246-254.

15 Hamer M, Stamatakis E, Mishra GD. Television- and Screen-Based Activity and Mental Well-Being in Adults. Am J Prev Med. 2010; 38(4): 375-380.

16 Goldberg DP, Gater R, Sartorius N, et al. The validity of two versions of the GHQ in the WHO study of mental illness in general health care. Psychol Med. 1997; 27(1): 191197.

17 Teychenne M, Ball K, Salmon J. Physical activity, sedentary behavior and depression among disadvantaged women. Health Educ Res. 2010; 25(4): 632-644.

18 Kraut R, Kiesler S, Boneva B, et al. Internet paradox revisited. J Soc Issues. 2002; 58: 49-74. 
19 Kraut R, Patterson M, Lundmark V, et al. Internet paradox. A social technology that reduces social involvement and psychological well-being? Am Psychol. 1998; 53(9): 1017-1031.

20 Bull FC, Adams EJ, Hooper PL. Well@Work: Promoting active and healthy workplaces final evaluation report. School of Sport and Exercise Sciences, Loughborough University. Available at: http://www.bhfactive.org.uk/homepageresources-and-publications-item/102/index.html; 2008.

21 Craig CL, Marshall AL, Sjostrom M, et al. International physical activity questionnaire: 12-country reliability and validity. Med Sci Sports Exerc. 2003; 35(8): 1381-1395.

22 Wareham NJ, Jakes RW, Rennie KL, et al. Validity and repeatability of the EPICNorfolk Physical Activity Questionnaire. Int J Epidemiol. 2002; 31(1): 168-174.

23 Sanchez-Villegas A, Ara I, Guillen-Grima F, et al. Physical activity, sedentary index, and mental disorders in the SUN Cohort Study. Med Sci Sports Exer. 2008; 40(5): 827834.

24 Teychenne M, Ball K, Salmon J. Physical activity, sedentary behavior and depression among disadvantaged women. Health Educ Res. 2010; 25(4): 632-644.

25 Marshall SJ, Biddle SJ, Gorely T, et al. Relationships between media use, body fatness and physical activity in children and youth: a meta-analysis. Int J Obes Relat Metab Disord. 2004; 28(10): 1238-1246.

26 Hamer M, Stamatakis E, Steptoe A. Dose-response relationship between physical activity and mental health: the Scottish Health Survey. Br J Sports Med. 2009; 43(14): $1111-1114$. 


\section{Table 1.}

Sample Characteristics with Reference to Total Non-Occupational Sitting Time ( $n=2707)$, \% unless otherwise stated.

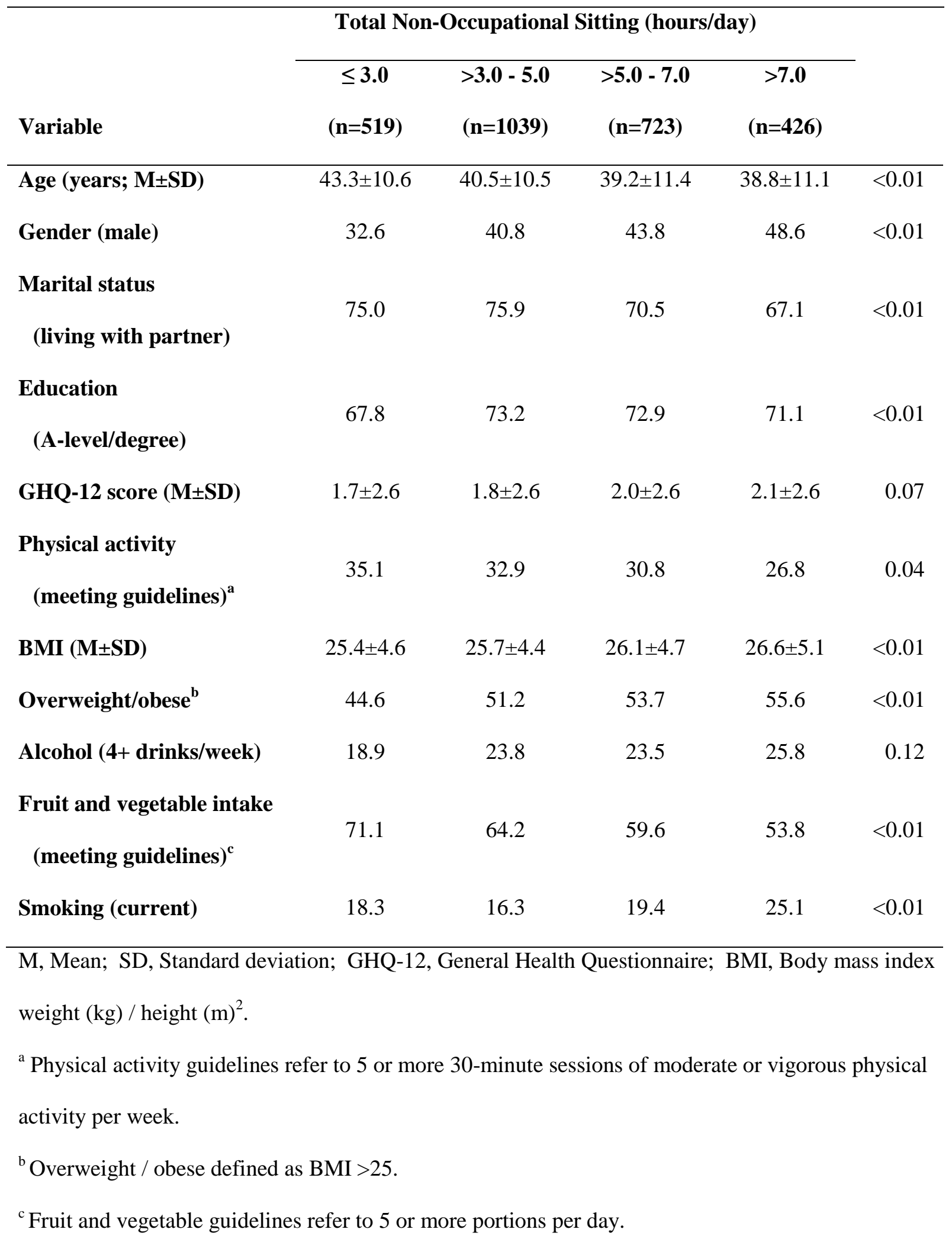


Table 2.

Association of Domain-Specific (mins/day) and Total Non-Occupational Sitting Time (hours/day) with GHQ-12 score (n=2707), $\beta$ (95\% CI) unless otherwise stated.

\begin{tabular}{|c|c|c|c|c|c|c|c|c|}
\hline \multirow[b]{2}{*}{ Variable } & \multicolumn{4}{|c|}{$\operatorname{Men}(n=1117)$} & \multicolumn{4}{|c|}{ Women $(n=1590)$} \\
\hline & $\begin{array}{l}\text { GHQ-12 } \\
(\mathrm{M} \pm \mathrm{SD})\end{array}$ & Model 1 & Model 2 & Model 3 & $\begin{array}{l}\text { GHQ-12 } \\
(\mathrm{M} \pm \mathrm{SD})\end{array}$ & Model 1 & Model 2 & Model 3 \\
\hline \multicolumn{9}{|l|}{ Total sitting (hrs/day) } \\
\hline Low $(<3.0)$ & $1.59 \pm 2.40$ & ref & ref & ref & $1.72 \pm 2.63$ & ref & ref & ref \\
\hline Medium (>3.0-5.0) & $1.70 \pm 2.55$ & $0.10(-0.51,0.71)$ & $0.16(-0.37,0.69)$ & $0.18(-0.37,0.72)$ & $1.90 \pm 2.67$ & $0.16(-0.13,0.45)$ & $0.03(-0.23,0.29)$ & $0.04(-0.22,0.30)$ \\
\hline High $(>5.0-7.0)$ & $1.92 \pm 2.57$ & $0.33(-0.24,0.90)$ & $0.29(-0.21,0.78)$ & $0.30(-0.20,0.80)$ & $2.03 \pm 2.65$ & $0.28(-0.02,0.59)$ & $0.15(-0.17,0.46)$ & $0.15(-0.17,0.46)$ \\
\hline Very high $(>7.0)$ & $1.66 \pm 2.31$ & $0.09(-0.45,0.63)$ & $-0.02(-0.54,0.49)$ & $-0.02,(-0.57,0.54)$ & $2.46 \pm 2.82$ & $0.70(0.39,1.02)$ & $0.50(0.21,0.80)$ & $0.51(0.22,0.80)$ \\
\hline p-trend & & 0.45 & 0.93 & 0.94 & & $<0.01$ & $<0.01$ & $<0.01$ \\
\hline \multicolumn{9}{|l|}{ TV (mins/day) } \\
\hline Low $(<60.0)$ & $1.46 \pm 2.04$ & ref & ref & ref & $1.75 \pm 2.58$ & ref & ref & ref \\
\hline Medium (>60.0-120.0) & $1.87 \pm 2.62$ & $0.40(0.15,0.65)$ & $0.41(0.11,0.71)$ & $0.38(0.08,0.68)$ & $1.98 \pm 2.64$ & $0.21(-0.20,0.62)$ & $0.21(-0.17,0.58)$ & $0.20(-0.16,0.56)$ \\
\hline High $(>120.0-180.0)$ & $1.79 \pm 2.60$ & $0.33(-0.10,0.76)$ & $0.37(-0.09,0.83)$ & $0.34(-0.12,0.79)$ & $1.96 \pm 2.70$ & $0.20(-0.15,0.56)$ & $0.10(-0.23,0.42)$ & $0.08(-0.22,0.39)$ \\
\hline Very high $(>180.0)$ & $1.70 \pm 2.47$ & $0.26(-0.07,0.59)$ & $0.32(-0.16,0.59)$ & $0.19(-0.18,0.56)$ & $2.29 \pm 2.88$ & $0.54(0.18,0.90)$ & $0.42(0.08,0.77)$ & $0.41(0.08,0.75)$ \\
\hline p-trend & & 0.42 & 0.56 & 0.60 & & $<0.01$ & 0.06 & 0.05 \\
\hline \multicolumn{9}{|l|}{ Computer (mins/day) } \\
\hline Low $(<30.0)$ & $1.57 \pm 2.3$ & ref & ref & ref & $1.88 \pm 2.67$ & ref & ref & ref \\
\hline
\end{tabular}




\begin{tabular}{|c|c|c|c|c|c|c|c|c|}
\hline Medium (>30.0-60.0) & $1.86 \pm 2.85$ & $0.28(-0.07,0.62)$ & $0.26(-0.00,0.53)$ & $0.28(0.05,0.52)$ & $1.88 \pm 2.54$ & $-0.06(-0.49,0.37)$ & $-0.01(-0.44,0.43)$ & $-0.01(-0.44,0.42)$ \\
\hline High $(>60.0-90.0)$ & $2.11 \pm 2.64$ & $0.53(-0.05,1.11)$ & $0.43(-0.13,1.00)$ & $0.42(-0.12,0.96)$ & $2.24 \pm 2.88$ & $0.33(-0.22,0.87)$ & $0.23(-0.32,0.78)$ & $0.22(-0.36,0.79)$ \\
\hline Very high (>90.0) & $1.88 \pm 2.43$ & $0.32(-0.01,0.65)$ & $0.25(0.01,0.49)$ & $0.28(0.03,0.53)$ & $2.62 \pm 2.88$ & $0.71(0.46,0.96)$ & $0.64(0.35,0.92)$ & $0.64(0.34,0.93)$ \\
\hline p-trend & & 0.03 & 0.02 & 0.01 & & $<0.01$ & $<0.01$ & $<0.01$ \\
\hline \multicolumn{9}{|l|}{ cial (mins/day) } \\
\hline Low $(<30.0)$ & $1.84 \pm 2.64$ & ref & ref & ref & $1.72 \pm 2.52$ & ref & ref & ref \\
\hline Medium (>30.0-60.0) & $1.75 \pm 2.54$ & $-0.11(-0.65,0.42)$ & $-0.10(-0.63,0.43)$ & $-0.10(-0.62,0.43)$ & $2.03 \pm 2.76$ & $0.29(0.03,0.55)$ & $0.32(0.04,0.61)$ & $0.33(0.03,0.63)$ \\
\hline High $(>60.0-90.0)$ & $1.67 \pm 2.30$ & $-0.18(-0.57,0.21)$ & $-0.22(-0.60,0.16)$ & $-0.22(-0.59,0.14)$ & $2.06 \pm 2.60$ & $0.32(-0.00,0.63)$ & $0.30(-0.01,0.62)$ & $0.32(0.02,0.62)$ \\
\hline Very high $(>90.0)$ & $1.66 \pm 2.39$ & $-0.16(-0.58,0.27)$ & $-0.25(-0.66,0.16)$ & $-0.24(-0.65,0.18)$ & $2.11 \pm 2.80$ & $0.35(-0.06,0.76)$ & $0.32(-0.05,0.69)$ & $0.34(-0.02,0.69)$ \\
\hline p-trend & & 0.32 & 0.09 & 0.12 & & 0.16 & 0.18 & 0.14 \\
\hline \multicolumn{9}{|l|}{ avel (mins/day) } \\
\hline Low $(<30.0)$ & $1.73 \pm 2.65$ & ref & ref & ref & $1.81 \pm 2.64$ & ref & ref & ref \\
\hline Medium (>30.0-60.0) & $1.59 \pm 2.41$ & $-0.14(-0.57,0.29)$ & $-0.13(-0.58,0.33)$ & $-0.13(-0.60,0.33)$ & $1.88 \pm 2.59$ & $0.03(-0.35,0.41)$ & $-0.01(-0.40,0.38)$ & $-0.01(-0.40,0.40)$ \\
\hline High (>60.0-90.0) & $2.11 \pm 2.67$ & $0.38(-0.06,0.82)$ & $0.30(-0.19,0.78)$ & $0.27(-0.18,0.72)$ & $1.84 \pm 2.60$ & $0.01(-0.47,0.49)$ & $-0.05(-0.59,0.48)$ & $-0.04(-0.58,0.49)$ \\
\hline Very high (>90.0) & $1.73 \pm 2.25$ & $-0.02(-0.50,0.46)$ & $0.03(-0.38,0.45)$ & $0.04(-0.37,0.45)$ & $2.48 \pm 2.91$ & $0.61(0.10,1.11)$ & $0.52(-0.01,1.05)$ & $0.51(-0.02,1.04)$ \\
\hline p-trend & & 0.58 & 0.46 & 0.43 & & 0.04 & 0.09 & 0.09 \\
\hline
\end{tabular}

$\beta$ values indicate mean difference in GHQ-12 score compared to reference (ref) group.

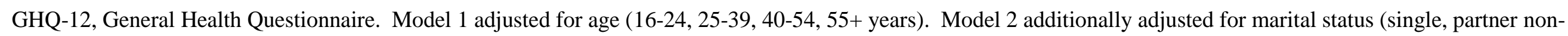

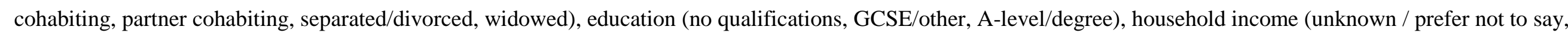


$<£ 20800, £ 20800-£ 41600,>£ 41600$ per year), smoking (never, former, current), BMI (<25, 25-30, >30), alcohol consumption (never, monthly or less, $2-4$ times/month, $2-$ 3 times/week, 4+ times/week), daily fruit and vegetable intake (not/meeting recommendations). Model 3 additionally adjusted for occupational (not at all physically active, not very physically active, fairly physically active, very physically active) and leisure-time physical activity level $(<60,60-180,180-300,>300$ minutes / week). 